\title{
Verzeichnis der R-Codes
}

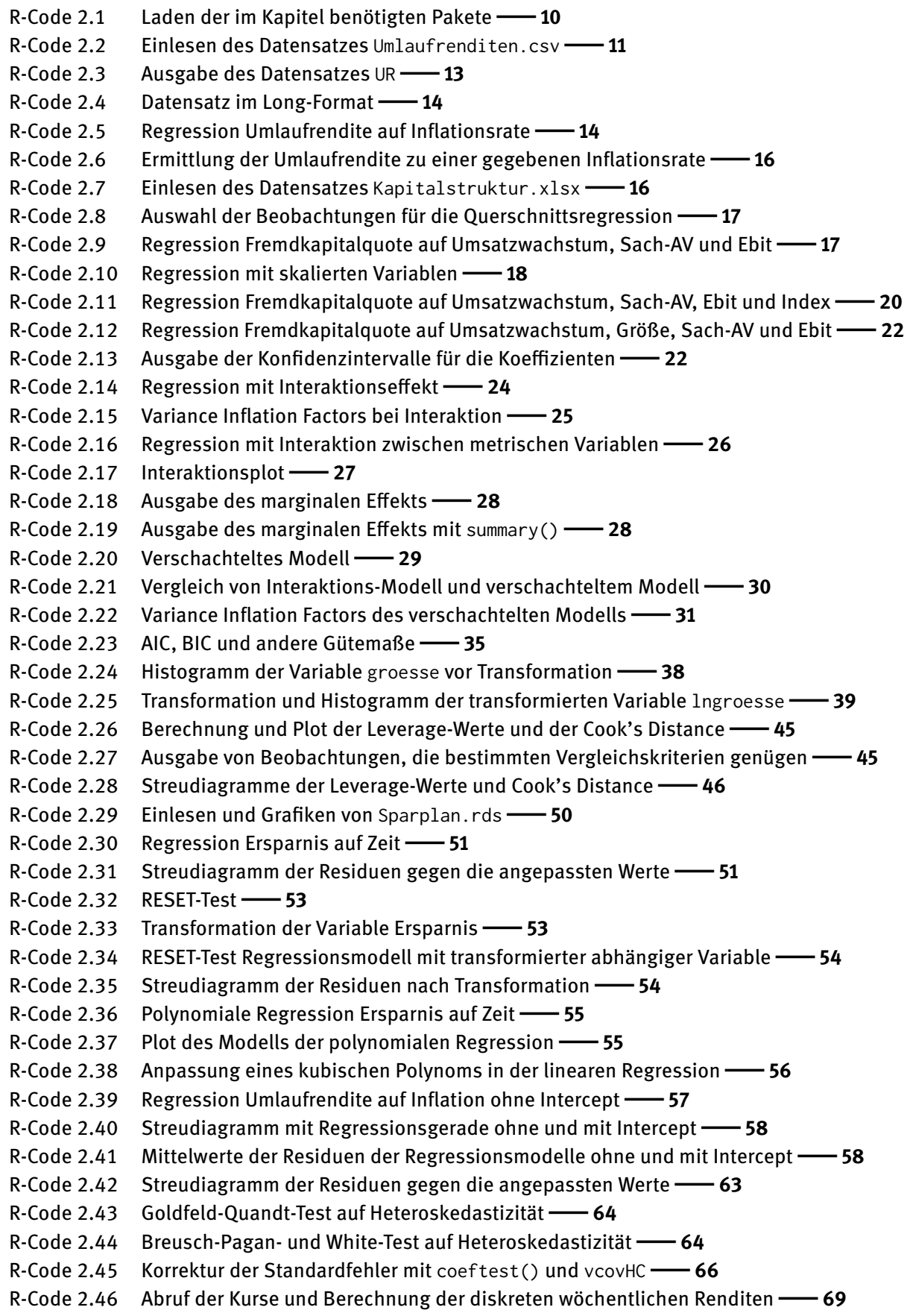


R-Code 2.47 Berechnung des Beta, Streudiagramm der Residuen gegen versetzte Residuen -70

R-Code 2.48 Korrelation zwischen den Residuen und den versetzten Residuen - 71

R-Code 2.49 Durbin-Watson-Test auf Autokorrelation $\longrightarrow 72$

R-Code 2.50

R-Code 2.51

R-Code 2.52

R-Code 2.53

R-Code 2.54

R-Code 2.55

R-Code 2.56

R-Code 2.57

R-Code 2.58

R-Code 2.59

Breusch-Godfrey-Test auf Autokorrelation höherer Ordnung -73

Korrektur der Standardfehler mit coeftest() und vcovHAC -74

F-Test mit korrigierten Varianz-Kovarianz-Schätzern - 75

Prüfung auf Multikollinearität mittels der Variance Inflation Factors -78

Vergleich der Mittelwerte des Umsatzwachstums bei unterschiedlichen Indices - 79

Shapiro- und Jarque-Bera-Test auf Normalverteilung der Residuen - $\mathbf{8 2}$

Histogramm mit Normalverteilungsdichte und QQ-Plot der Residuen $-\mathbf{8 3}$

Ausgabe verschiedener diagnostischer Grafiken - 84

Modellselektion mittels der step()-Funktion - $\mathbf{8 8}$

Kreuzvalidierung für die Prognose -91

R-Code 3.1

Laden der im Kapitel benötigten Pakete - 96

R-Code 3.2

R-Code 3.3

Laden des Datensatzes CreditSpreads.rds -96

R-Code 3.4

Umformen des Datensatzes — 97

R-Code 3.5

Vergleichende Liniendiagramme -97

Pooled Regression — 99

Lineare Regression - $\mathbf{1 0 0}$

R-Code 3.6

Streudiagramm Zinsspread gegen Nominalzins - 100

R-Code 3.7

Fixed Effects Model -103

R-Code 3.8

Ausgabe der fixen Effekte - 104

R-Code 3.10

R-Code 3.11

R-Code 3.12

R-Code 3.13

R-Code 3.14

R-Code 3.15

R-Code 3.16

Vergleich des Fixed Effects Model mit einer linearen Regression - 104

F-Test des Fixed Effects Model -105

Random Effects Model - 107

Lagrange-Multiplier-Test auf zufällige Effekte - 108

Hausman-Test -109

R-Code 3.17

Ausgabe der Koeffizienten und der fixen Effekte - $\mathbf{1 1 0}$

R-Code 3.18

Fixed Effects Model: Tests auf Heteroskedastizität - 112

Fixed Effects Model: Tests auf Normalverteilung der Residuen - 112

R-Code 3.19

Fixed Effects Model: Tests auf Autokorrelation - 113

R-Code 3.20

Fixed Effects Model: Korrektur der Standardfehler - 115

R-Code 3.21

Fixed Effects Model: Tests auf Querschnittskorrelation — 115

R-Code 3.21
R-Code 3.22

Fixed Effects Model: Korrektur der Standardfehler bei Querschnittskorrelation - 116

Fixed Effects Model: Prüfung auf Multikollinearität - 117

R-Code 4.1

Laden der im Kapitel benötigten Pakete - 124

R-Code 4.2

Einlesen des Datensatzes Kreditstatus.rds - 127

R-Code 4.3

Logistische Regression ausfall alter $-\mathbf{1 2 8}$

R-Code 4.4

R-Code 4.5

Ausgabe des transformierten Koeffizienten -129

R-Code 4.6

Logistische Regression ausfall wohnen - 129

R-Code 4.7

Änderung der Referenzkategorie $-\mathbf{1 3 0}$

R-Code 4.8

Interpretation der marginalen Effekte - $\mathbf{1 3 1}$

R-Code 4.9

Plot der mittleren marginalen Effekte (ohne Interaktion) - 133

R-Code 4.10

Ausgabe der Devianz — 135

R-Code 4.11

Differenz zwischen Nulldevianz und Modelldevianz - 135

R-Code 4.12

Likelihood-Quotienten-Test für ein logistisches Regressionsmodell - 136

Wald-Test für die Koeffizienten — 137 
R-Code 4.13 Likelihood-Quotienten-Test für einen Koeffizienten — 139

R-Code 4.14 Interpretation der marginalen Effekte mit summary () $-\mathbf{1 4 0}$

R-Code 4.15 Ausgabe der marginalen Effekte für al ter bedingt durch alter $\longrightarrow \mathbf{1 4 0}$

R-Code 4.16 Ausgabe der marginalen Effekte für al ter bedingt durch betrag - 141

R-Code 4.17 Logistische Regression mit Interaktion zwischen metrischen Variablen - 143

R-Code 4.18 Plot des mittleren marginalen Effekts bei Interaktion - 143

R-Code 4.19 Plot der modellierten Wahrscheinlichkeiten - 144

R-Code 4.20 Ausgabe der marginalen Effekte in einem Logit-Modell mit Interaktion — 144

R-Code 4.21 Einflussreiche Beobachtungen: Berechnung und Plot der Statistiken -148

R-Code 4.22 Einflussreiche Beobachtungen im Datensatz — 148

R-Code 4.23 Ausgabe wichtiger diagnostischer Größen mit augment () $-\mathbf{1 5 0}$

R-Code 4.24 Test auf nichtlinearen Zusammenhang - 151

R-Code 4.25 Streudiagramm einkommen gegen Logit $\eta-152$

R-Code 4.26 Variance Inflation Factors - 153

R-Code 4.27 Likelihood-Quotienten-Test zum Vergleich von Modellen - 154

R-Code 4.28 Vergleich von Modellen über AIC und BIC — 155

R-Code 4.29 Berechnung der prognostizierten Ausfälle — 157

R-Code 4.30 Ausgabe der Konfusionsmatrix - 157

R-Code 4.31 Ausgabe der Konfusionsmatrix und verschiedener anderer Gütekriterien — 159

R-Code 4.32 Ausgabe von Recall und Präzision -160

R-Code 4.33 ROC-Kurve - 161

R-Code 4.34 Ermittlung des AUC $-\mathbf{1 6 2}$

R-Code 4.35 Ermittlung des optimalen Cutpoints - 163

R-Code 4.36 Berechnung und Plot der Liftkurve -164

R-Code 4.37 Anteil überdurchschnittlicher Liftwerte - 165

R-Code 4.38 Ermittlung der Liftwerte für einzelne Quantile — 165

R-Code 5.1 Laden der im Kapitel benötigten Pakete - 168

R-Code 5.2 Einlesen und Vorbereitung des Datensatzes - $\mathbf{1 7 0}$

R-Code 5.3 Berechnung und Ausgabe des Klassifikationsbaums - 171

R-Code 5.4 Ausgabe der Kategorien der Variable Index - $\mathbf{1 7 2}$

R-Code 5.5 Plot des Klassifikationsbaums - 172

R-Code 5.6 Ausgabe der mit einem Klassifikationsbaum modellierten Werte - 173

R-Code 5.7 Fehlklassifikationsrate des Klassifikationsbaums - $\mathbf{1 7 3}$

R-Code 5.8 Fehlklassifikationsrate der Diskriminanzanalyse - $\mathbf{1 7 4}$

R-Code 5.9 3-fache Kreuzvalidierung: Aufteilung in Trainings- und Testdaten -174

R-Code 5.10 Kreuzvalidierung des Klassifikationsbaums - $\mathbf{1 7 5}$

R-Code 5.11 Klassifikationsbaum mit numerischen Variablen als Prädiktoren - $\mathbf{1 7 5}$

R-Code 5.12 Überangepasster Klassifikationsbaum — 177

R-Code 5.13 Beschneidung des Klassifikationsbaums - $\mathbf{1 7 8}$

R-Code 5.14 Plot der Komplexitätsparameter eines Baums - 179

R-Code 5.15 Berechnung eines Regressionsbaums - 181

R-Code 5.16 Plot des Regressionsbaums - 182

R-Code 5.17 Prognose mittels eines Regressionsbaums - 182

R-Code 5.18 MSE des Regressionsbaums - 184

R-Code 5.19 MSE des linearen Regressionsmodells KSQ.1m — 184

R-Code 5.20 Mittelwert der Residuen - Regressionsbaum und lineares Modell im Vergleich - 184

R-Code 5.21 3-fache Kreuzvalidierung des Regressionsbaums und des linearen Modells - 185

R-Code 5.22 Komplexitätstabelle -186 
R-Code 5.23 Modellierung eines Random Forest für die Klassifikation mit randomForest() - 190

R-Code 5.24 Ausgabe der Fehlklassifikationsrate des Random-Forest-Modells - 190

R-Code 5.25 Modellierung eines Random Forest für die Klassifikation mit cforest() 191

R-Code 5.26 Konfusionsmatrix und weitere Klassifikationskennzahlen - 191

R-Code 5.27 Modellierung eines Random Forest für die Regression - 194

R-Code 5.28 Prognose mit Random Forest - 196

R-Code 5.29 Random Forest: Wichtigkeit der Variablen - 198

R-Code 5.30 Tabellarische Darstellung der Wichtigkeit der Variablen mit randomForest -199

R-Code 5.31 Lineares Modell mit skalierten Variablen — 199

R-Code 5.32 Wichtigkeit der Variablen mit cforest () - $\mathbf{2 0 0}$

R-Code 5.33 Ausgabe der absteigend sortierten Variablenwichtigkeit $-\mathbf{2 0 0}$

R-Code 6.1 Laden der im Kapitel benötigten Pakete - 208

R-Code 6.2 Einlesen und Vorbereiten des Datensatzes Kapitalstruktur.xlsx 209

R-Code 6.3 Entfernen der Variable $\mathrm{fkq} \longrightarrow \mathbf{2 0 9}$

R-Code 6.4 Plot der Korrelationsmatrix - 209

R-Code 6.5 Kaiser-Maier-Olkin-Kriterium und Bartlett-Test - 210

R-Code 6.6 MSA-Kriterien der einzelnen Variablen - 211

R-Code 6.7 Erklärte Varianz der Hauptkomponenten - 212

R-Code 6.8 Durchführung der Hauptkomponentenanalyse ohne Rotation - 213

R-Code 6.9 Durchführung der Hauptkomponentenanalyse mit Varimax-Rotation - 215

R-Code 6.10 Biplot der ersten beiden rotierten Hauptkomponenten - $\mathbf{2 1 6}$

R-Code 6.11 Erstellung eines Datensatzes mit Einbindung der Scores für weitere Analysen - 217

R-Code 6.12 Lineare Regression der Fremdkapitalquote auf die vier Hauptkomponenten - 218

R-Code 6.13 Korrektur der Standardfehler mit coeftest() - 218

R-Code 7.1 Laden der im Kapitel benötigten Pakete - 221

R-Code 7.2 Abruf und Plot der Kursdaten des DAX - 222

R-Code 7.3 Korrelogramme der quadrierten DAX-Renditen - 225

R-Code 7.4 Scheinregression zweier Random Walks - 229

R-Code 7.5 Phillips-Ouliaris-Test bei Scheinregression - 230

R-Code 7.6 1000-fache Wiederholung der Regression von zwei Random Walks - 230

R-Code 7.7 Stationaritätstest am Beispiel eines Random Walks — 234

R-Code 7.8 Stationaritätstest nach Differenzbildung - 235

R-Code 7.9 Laden des Datensatzes Adidas.xlxs -241

R-Code 7.10 Umwandlung in ein ts-Objekt -242

R-Code 7.11 Stationaritätstests für die Zeitreihe ADI - 242

R-Code 7.12 Differenzbildung und Stationaritätstests $-\mathbf{2 4 3}$

R-Code 7.13 Plot der ersten Differenz der Zeitreihe ADI - $\mathbf{2 4 3}$

R-Code 7.14 ACF und PACF der ersten Differenz - 244

R-Code 7.15 ARIMA(4,1,0)-Modell: Modellierung und serielle Unabhängigkeit der Residuen - 245

R-Code 7.16 ARIMA(4,1,0)-Modell: QQ-Plot der Residuen und Test auf Normalverteilung - 245

R-Code 7.17 Verschiedene diagnostische Plots - 247

R-Code 7.18 Automatische Schätzung der Modellparameter eines ARIMA-Modells - 248

R-Code 7.19 Forecast aus einem ARIMA-Modell -249

R-Code 7.20 Test auf einen ARCH-Effekt $\longrightarrow 255$

R-Code 7.21 Simulation eines ARCH(4)- und eines GARCH(1,3)-Prozesses - 257

R-Code 7.22 SAP-Renditen abrufen und stetige Renditen berechnen - 258

R-Code 7.23 Liniendiagramme der SAP-Kurse und der stetigen Renditen - 259 
R-Code 7.24 ACF und PACF der Renditen und quadrierten Renditen von SAP $-\mathbf{2 6 0}$

R-Code 7.25 McLeod-Li-Test auf Heteroskedastizität - 261

R-Code 7.26 Extended-ACF-Diagramm der quadrierten Renditen — 261

R-Code 7.27 Modellierung eines GARCH(1,1)-Modells am Beispiel SAP - 262

R-Code 7.28 Liniendiagramm der Residuen des GARCH(1,1)-Modells - 263

R-Code 7.29 Plot der Renditen und der modellierten Varianz -264

R-Code 7.30 Prognose der Volatilitäten aus einem GARCH(1,1)-Modell — 264

R-Code 7.31 Vergleich verschiedener GARCH-Modelle - 266

R-Code 8.1 Laden der im Kapitel benötigten Pakete - $\mathbf{2 7 8}$

R-Code 8.2 Variablen für die Tickersymbole anlegen - $\mathbf{2 7 9}$

R-Code 8.3 Abruf der Aktienkurse der Banken und Berechnung der stetigen Renditen $-\mathbf{2 8 0}$

R-Code $8.4 \quad$ Normale und abnormale Renditen berechnen - $\mathbf{2 8 0}$

R-Code 8.5 Tests zur Überprüfung auf das Vorliegen abnormaler Renditen - $\mathbf{2 8 1}$

R-Code 8.6 Überprüfung und Ausgabe der CAAR - 282

R-Code 8.7 Vergleich der AAR von britischen und nicht-britischen Banken $-\mathbf{2 8 3}$ 
